\title{
Enhanced processing of a lost language: Linguistic knowledge or linguistic skill?
}

\author{
Jiyoun Choi ${ }^{1,2}$, Mirjam Broersma ${ }^{2,3}$, Anne Cutler ${ }^{2,4}$ \\ ${ }^{1}$ Hanyang Phonetics and Psycholinguistics Laboratory, Hanyang University, Korea \\ ${ }^{2}$ Max Planck Institute for Psycholinguistics, The Netherlands \\ ${ }^{3}$ Centre for Language Studies, Radboud University Nijmegen, The Netherlands \\ ${ }^{4}$ MARCS Institute, University of Western Sydney, Australia \\ jiychoi@hanyang.ac.kr, m.broersma@let.ru.nl, a.cutler@uws.edu.au
}

\begin{abstract}
Same-different discrimination judgments for pairs of Korean stop consonants, or of Japanese syllables differing in phonetic segment length, were made by adult Korean adoptees in the Netherlands, by matched Dutch controls, and Korean controls. The adoptees did not outdo either control group on either task, although the same individuals had performed significantly better than matched controls on an identification learning task. This suggests that early exposure to multiple phonetic systems does not specifically improve acoustic-phonetic skills; rather, enhanced performance suggests retained language knowledge.

Index Terms: discrimination, Korean, Japanese, phoneme perception, international adoptees
\end{abstract}

\section{Introduction}

Some people acquire and use only one language, many people acquire and use more than one language, and then there are people who begin to acquire one language but must then switch to another. Of the latter, international adoptees form an interesting case since they can provide evidence on the earliest stages of learning about a language, and the memories formed as a result of such learning, independently of later experience. An increasingly large body of research on adoptee populations [1-9] has in general shown that they usually receive no further input in the language they originally heard $[2,3]$, and retain essentially no active accessible knowledge of it [6-9].

Nonetheless, adoptees do appear to retain phonological experience of the language once heard but now lost. Adult Korean adoptees in the Netherlands trained to identify Korean fortis, lenis and aspirated stop consonants significantly outperformed Dutch control participants given the same training [8]; also, brain scans of teenage Chinese-born adoptees in Canada showed that Chinese speech activated brain areas also active in Chinese listeners [9]. Early phonological experience thus leaves traces even if the language is unused for decades.

However, given that adoptees' first-language knowledge is lost to their awareness, the nature of these advantages warrants investigation. In [8], identification performance was measured in a forced-choice categorization task (with the above-named three alternatives). It is possible that adoptees' advantages primarily lay in acoustic-phonetic processing here; adoptees may have formed more accurate mental representations of the target phonetic categories, and/or might have been better (than the Dutch controls) at assigning the target sounds to these categories. Early experience with two quite different sets of phonetic categories may enhance the required phonetic skill.
Such an interpretation, based only on enhancement of the ability to process phonetic structure, has several interesting implications; first, it does not necessarily entail that languagespecific knowledge is retained (only general skill in language processing), and second, it implies that better ability should be seen wherever it is used (i.e., not only in an original language). The present study further explores the nature of adoptees' processing advantages by addressing these implications. With the same participants as in [8], we examine whether enhanced performance generalizes across task type and across language.

Instead of testing identification, we tested discrimination of the same three Korean (fortis, lenis and aspirated) stop consonants. It has been argued $[10,11]$ that identification and discrimination tasks tap into different perceptual processes that discrimination taps lower acoustic-phonetic processing, while identification taps into the level at which phonemic categories play a role. Thus correlations between identification and discrimination performance are weak [10], and listeners who succeed in assigning sounds to phonetic categories in identification can still fail to discriminate the same sounds [11]

We also tested a difficult contrast from Japanese, namely a three-way length discrimination in vowel-consonant-vowel (VCV) sequences. Like the three-way Korean stop contrast, this has no equivalent in either Dutch or Korean. Thus each of these contrasts, stop and length, will be difficult to distinguish for native listeners of both Dutch and Korean [12, 13, 14].

Finally, this study also tested Korean adult control listeners, again matched in age to the adoptee participants.

Thus the study addresses both the question whether language-specific knowledge is retained, and the question whether language-processing skill is generally enhanced. If the adoptees perform better than the Dutch control participants at discriminating the Korean stops, as they did at identifying the same stops in [8], such generalization may indicate presence of stored knowledge of Korean, or better skill in processing. Secondary questions are then how much knowledge is retained (do adoptees approach the performance level of Korean native listeners?), and whether combinations of fortis, lenis, and aspirated targets differ for any group in the difficulty with which they are discriminated (are there only intrinsic acoustic difficulty differences, for all groups, or language-specific effects showing specific knowledge in Korean controls, then potentially in adoptees). Further, if adoptees have generally enhanced phonetic processing skills, then we expect that they may outperform both the Dutch and the Korean control groups in dealing with the unfamiliar Japanese length contrast. If the adoptees do not outperform controls in discrimination, though, then they surely do not have better phonetic processing skills. 


\section{Method}

\subsection{Participants}

Participants were 29 Korean-born adult Dutch speakers ( $M_{\text {age }}$ $=31.66$ years), who had been adopted at between 3 and 69 months of age, plus 54 control participants: 29 Dutch-born $\left(M_{\text {age }}=32.03\right.$ years $)$, and 25 Korean-born $\left(M_{\text {age }}=29.56\right.$ years $)$.

\subsection{Materials}

\subsubsection{Korean}

The tested contrast was a Korean three-way stop contrast of fortis, lenis, and aspirated stops. There were three sets of stimuli, differing in place of articulation; alveolar, bilabial, and velar. For each of these three sets, three minimal triplets of CVCV Korean pseudo-words were created, giving 27 items in all (Table 1). Within each triplet, items varied only in wordinitial fortis, lenis, or aspirated stops; the following vowel was [a], [i], or [u], and the final syllable always [mi].

Table 1. Nine Korean minimal triplets.

\begin{tabular}{llll}
\hline Place of articulation & Fortis & Lenis & Aspirated \\
\hline \multirow{3}{*}{ Alveolar } & {$\left[\mathrm{t}^{*}\right.$ ami $]$} & {$[$ tami $]$} & {$\left[\mathrm{t}^{\mathrm{h}}\right.$ ami $]$} \\
& {$\left[\mathrm{t}^{*}\right.$ imi $]$} & {$[$ timi $]$} & {$\left[\mathrm{t}^{\mathrm{h}}\right.$ imi $]$} \\
& {$\left[\mathrm{t}^{*}\right.$ umi $]$} & {$[$ tumi $]$} & {$\left[\mathrm{t}^{\mathrm{h}} \mathrm{umi}\right]$} \\
\hline \multirow{3}{*}{ Bilabial } & {$\left[\mathrm{p}^{*}\right.$ ami $]$} & {$[$ pami $]$} & {$\left[\mathrm{p}^{\mathrm{h}}\right.$ ami $]$} \\
& {$\left[\mathrm{p}^{*}\right.$ imi $]$} & {$[$ pimi $]$} & {$\left[\mathrm{p}^{\mathrm{h}} \mathrm{imi}\right]$} \\
& {$\left[\mathrm{p}^{*}\right.$ umi $]$} & {$[$ pumi $]$} & {$\left[\mathrm{p}^{\mathrm{h}} \mathrm{umi}\right]$} \\
\hline \multirow{3}{*}{ Velar } & {$\left[\mathrm{k}^{*}\right.$ ami $]$} & {$[$ kami $]$} & {$\left[\mathrm{k}^{\mathrm{h}}\right.$ ami $]$} \\
& {$\left[\mathrm{k}^{*}\right.$ imi $]$} & {$[$ kimi $]$} & {$\left[\mathrm{k}^{\mathrm{h}}\right.$ imi $]$} \\
& {$\left[\mathrm{k}^{*}\right.$ umi $]$} & {$[$ kumi $]$} & {$\left[\mathrm{k}^{\mathrm{h}}\right.$ umi $]$} \\
\hline
\end{tabular}

A female native speaker of Korean recorded multiple tokens of all 27 items using a Sennheiser microphone in a soundproof booth and sampling at $44.1 \mathrm{kHz}$. Four test tokens of each item were then selected, giving 108 tokens in all.

72 pairs were constructed: 36 Same pairs, 36 Different pairs. Of the Different pairs, 18 were experimental pairs that differed in the crucial stops, and the other 18 were filler pairs differing in the vowels in the first syllable. For the Different experimental pairs, each consonant type was paired with every other consonant type (i.e., fortis-lenis, fortis-aspirated, lenisaspirated) in both orders. Likewise, for Different filler pairs, each first vowel was paired with every other first vowel (i.e., [a]-[i], [a]-[u], [i]-[u]) in both orders. Same pairs always consisted of two different recorded tokens of the same item.

\subsubsection{Japanese}

The test contrast was a Japanese three-way length distinction of (1) an initial short vowel plus a long consonant (henceforth: Geminate), (2) an initial short vowel plus a short consonant (henceforth: Singleton), and (3) an initial long vowel plus a short consonant (henceforth: Long Vowel). Three triplets of VCV Japanese pseudo-words contrasting these targets in the initial syllable were created (Table 2), i.e. nine items in all. The first vowel (either short or long) could be [a], [i] or [u], the consonant (either singleton or geminate) was always $[\mathrm{f}],{ }^{1}$ and the second vowel was always short and always [a]. The nine items were recorded, by a female native speaker of Japanese, and test pairs were constructed, in both cases in the same manner as for the Korean test contrast.

\footnotetext{
1 The fricative occurs only as an allophone of $/ \mathrm{h} /$ [15] and as marginal consonants [16] in Japanese.
}

Table 2. Three Japanese triplets.

\begin{tabular}{lll}
\hline Geminate & Singleton & Long Vowel \\
\hline$[\mathrm{af:a}]$ & {$[\mathrm{afa}]$} & {$[\mathrm{a}: \mathrm{fa}]$} \\
{$[$ if:a] } & {$[\mathrm{ifa}]$} & {$[\mathrm{i}: \mathrm{fa}]$} \\
{$[\mathrm{uf:a}]$} & {$[\mathrm{ufa}]$} & {$[\mathrm{u}: \mathrm{fa}]$} \\
\hline
\end{tabular}

\subsection{Procedure}

In each discrimination task, all pairs were presented in random order. Participants were informed that they would hear two words, and were asked to determine whether the two were the same or different. They responded by pressing one of two keys on the computer keyboard. There was no response time-out.

Both the Korean and the Japanese discrimination tasks were administered in the course of the identification training sessions undertaken by the adoptees and Dutch controls over a period of 11 days. This training was to identify the three Korean stops (at the alveolar place of articulation only: [t*ami]-[tami]-[ $\mathrm{t}^{\mathrm{h}}$ ami]) in a three-alternative forced-choice identification task with feedback. These two groups were tested on these tasks before and after training and also midway through the training. Testing for these two trained groups took place at the participants' own homes or workplaces. The Korean control group was tested once only, at Hanyang University, Seoul, South Korea. Five members of this latter group had studied Japanese, and their Japanese discrimination task results were excluded from analysis.

\section{Results}

\subsection{Korean}

\subsubsection{Overall Sensitivity: Adoptees vs. Dutch Controls}

As a measure of perceptual sensitivity, $d^{\prime}$ (d-prime) values were used as the dependent variable [17]. $d^{\prime}$ was calculated over performance on both Same and Different experimental pairs, separately per participant, place of articulation (alveolar, bilabial, velar), and test (pre-test, midway test, post-test).

An ANOVA on $d^{\prime}$ comparing adoptees and Dutch controls revealed no significant differences: no main effect of Group and no significant interaction of Group and Test. Group and Place interacted $(F[2,112]=4.9, p<.05)$, but follow-up analyses showed no group effect at any place of articulation. A main effect of Test $(F[2,112]=6.8, p<.05)$ showed that participants' performance was better at the midway than at the pre-test $(F[1,56]=10.5, p<.05)$, but there was no difference between the midway test and the post-test, suggestive of an early increase in task aptitude but no consistent growth of sensitivity.

\subsubsection{Comparison with Korean Controls}

The results of the adoptees and Dutch controls at the post-test were compared to those of the Korean control participants. Analyses were conducted with $d^{\prime}$ as the dependent variable, and also with proportions of correct responses for the Different experimental pairs. As Figure1 clearly shows, Korean controls (who participated in only a single test session) exhibited high accuracy, and significantly outperformed both the adoptees and the Dutch controls; there was no indication that either the adoptees or the Dutch control participants approached Korean native-like performance, despite their identification training. 


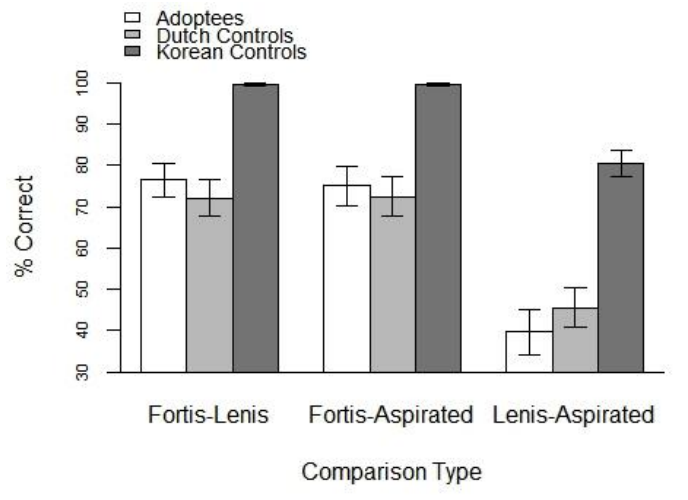

Figure 1: Percentage correct (with standard error) for the three Korean stop comparisons (collapsed over place of articulation), separately for adoptees and Dutch controls at post-test, and for Korean controls.

An ANOVA, with $d^{\prime}$ as dependent variable, over Place and Group showed, as expected, a significant main effect of Group $(F[2,80]=84.2, p<.05)$. Place and Group interacted $(F[4,160]$ $=10.2, p<.05) ; t$-tests with Bonferroni correction showed that the native Koreans performed better $(p s<.001)$ than adoptees and Dutch controls at all three places of articulation. Analyses on proportions of correct responses now also including Comparison type (fortis vs. lenis, fortis vs. aspirated, lenis vs. aspirated) and therefore on Different experimental pairs only revealed significant main effects of Group $(F 1[2,80]=24.3$, $p<.05 ; \quad F 2[2,90]=191.7, \quad p<.05)$ and of Comparison type $(F 1[2,160]=81.9, p<.05 ; F 2[2,45]=50.0, p<.05)$, plus a threeway interaction $(F 1[8,320]=3.9, p<.05 ; F 2[8,90]=2.1, p<.05)$; $t$-tests with Bonferroni correction confirmed that Koreans outperformed adoptees and Dutch controls in all comparisons.

\subsubsection{Relative Difficulty of Comparison types}

The three comparison types were compared with analyses of proportion correct across Different experimental pairs only. For adoptees and Dutch controls, a significant main effect of Comparison type appeared $(F 1[2,112]=71.5, p<.05 ; F 2[2,45]=$ 31.6, $p<.05$ ); follow-up analyses showed that the lenisaspirated comparisons received significantly fewer correct responses than the other two comparison types, which did not differ. In line with the $d^{d}$ analysis, there was no significant main effect of Group and no significant interaction between Group and Test, or between Group and Comparison type. All other effects on this subset of the data were also largely in line with the $d$ analysis performed on the full data set. For the Korean native listeners the result was the same $(F 1[2,48]$ $=38.8, p<.05 ; F 2[2,45]=38.6, p<.05)$. Thus, discrimination of the lenis vs. the aspirated stop was more difficult than the other two comparison types, for all listener groups equally.

The Korean discrimination test with the three listener groups has thus provided a clear negative answer to all the questions it addressed: the adoptees did not outperform the Dutch control group in the way that they had done in learning to perform identifications of the Korean stops; they did not reach anywhere near Korean native performance; and there was no language-specific difference across the comparisons in discrimination difficulty, only a language-general (and hence putatively acoustically based) difference.

\subsection{Japanese}

\subsubsection{Overall Sensitivity: Adoptees vs. Dutch Controls}

An ANOVA on $d^{\prime}$ across participants with the variables Test and Group revealed no significant Group effect or interaction of Test and Group; that is, the adoptees and Dutch controls did not differ in discrimination of the Japanese contrast.

\subsubsection{Comparison with Korean Controls}

The results of the Korean controls were compared to the pretest results of the adoptees and the Dutch controls. First, an ANOVA on $d^{\prime}$ across participants showed no significant effect of Group. Analyses with proportions of correct responses (including Comparison type, hence Different experimental pairs only) also showed that the three groups performed similarly, as can be seen in Figure2. There was a significant overall interaction of Group by Comparison type ( $F[4$, $150]=3.2, p<.05$ ); however $t$-tests with Bonferroni correction showed no group differences to be significant. Thus the three groups performed similarly on all comparisons.

Thus the Japanese discrimination test has also produced a clear answer to the question it was designed to address, and once again the answer is negative. There was no advantage in performing this discrimination task for any group, and in particular, the adoptees did not show any evidence of added processing skill in comparison to the other two groups.

It should be noted, however, that performance of all groups was high for the comparisons involving a long vowel. Previous work [13, 14] had revealed the Japanese geminate contrast to be hard for Dutch and Korean listeners, and all groups found this comparison hard if no long vowel was involved. However Korean and Dutch (like indeed probably all languages) require listeners to discriminate length differences of some kind, whether for detecting phrase boundaries or stress differences or phonemic differences. Length differences of long versus short vowels are even perceptible by quite young infants [18]. Thus we cannot exclude the possibility that the vowel length contrasts were too easy to reveal processing ability differences between our participants, whereas more variability might have been shown with a more difficult unfamiliar discrimination challenge.

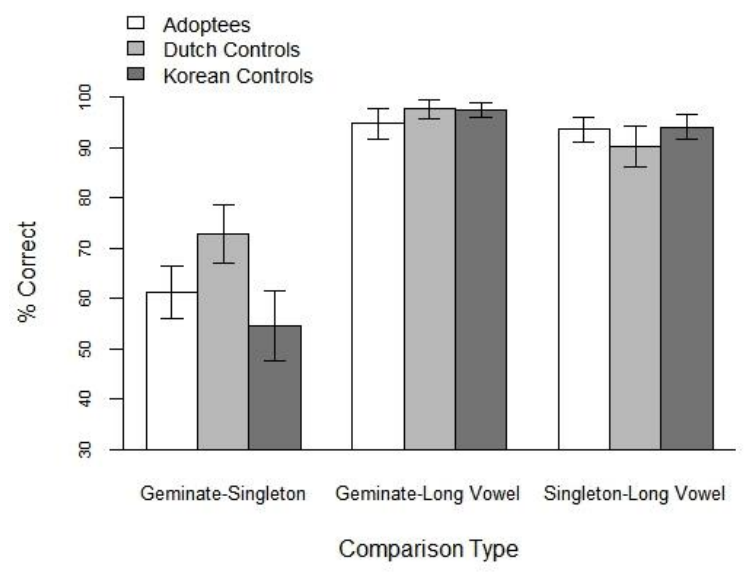

Figure 2: Percentage correct (with standard error) for the three Japanese length-based comparisons, for the adoptees and the Dutch controls at pre-test, and for the Korean control participants. 


\section{Discussion}

The present study had the purpose of investigating whether adult adoptees, who as children had first been exposed to Korean, would outperform Dutch control participants on discrimination of Korean stop contrasts, and if so, whether they would also outperform control groups on another difficult discrimination task with which they had never had any prior experience. The results provided a highly consistent picture. In the Korean discrimination study, neither an overall analysis of $d$ ' scores across the full data set (i.e., Same and Different experimental trials), nor analyses of proportions correct for the Different experimental trials only, provided any evidence of adoptees performing better than either control groupcompared with the Dutch controls, they were no better, and compared with the native Korean controls, they performed significantly less well. In the Japanese discrimination study, again no evidence of better performance by the adoptee group appeared, and here in fact all three groups (adoptees and the two control groups) performed quite well and did not differ significantly, with the only difficult condition being, for all three participant groups, comparing a geminate with a short consonant in the context of short vowels.

These results shed important light on the question of what experience can be retained and can exercise a lasting influence upon individuals who are first exposed to one language and then removed from that initial language environment and presented instead with input in a completely different language. The growing belief in language studies that such populations have interesting insights to offer [1-9] has recently produced clear evidence, both behavioral [8] and neuropsychological [9], of some retained processing benefit of this initial language exposure, even though it may have been relatively brief (a year or even less) and even though no input in the first language has since been received and that language is effectively lost. Similar evidence of processing benefits has been reported [1922] for heritage language users whose experience generally is of substantial reduction (rather than complete cutoff) in exposure to a family language heard in early childhood.

It is still unknown, however, whether adoptees' processing benefit actually involves language-specific knowledge. The contrasting hypothesis tested in the present study was that the benefit reflects, rather, an increase in phonetic processing skill consequent upon the need to deal with two potentially very different phonetic systems very early in life. This hypothesis must be considered to have forfeited much of its credibility in the light of the present results, in which a previously observed [8] adoptee processing benefit for Korean stop contrasts failed to generalize either across task or across test language.

The observation in [8] came from the same participants tested in the present study. The results presented here suggest that these individuals do not possess especially enhanced skills in phonetic processing. However, in a different task, which required them to learn to identify the Korean contrasts, they did outperform the Dutch controls. In particular, their speed of learning the successful identification outstripped that of the matched control group. This could be evidence of residual retained knowledge of the stop contrasts in question (which do not occur in their adopted language, Dutch). If so, the result is fully compatible with a retained-knowledge interpretation of the similarly recent finding [9] that Chinese tone distinctions activate language processing areas in the brains of Chineseborn but now French-speaking teenagers.
Why then does such retained knowledge lead to an observable benefit in an identification task, but not in a discrimination task such as that used in the present study? We suggest that this asymmetry in the results provides further evidence that the two types of task tap into very different perceptual processes, as previously proposed [10, 11, 13]. Identification, by its very nature, involves a categorization element and cannot be performed without knowledge of the categories in question. On the other hand, discrimination (in its simplest form, same-different judgment) can in principle be performed at a purely acoustic level, without engaging any language-specific knowledge at all. Thus a study [23] in which same-different judgments were made on Cantonese syllable pairs differing in tones 1 vs. 2 produced almost exactly equal performance from Cantonese listeners $(91.8 \%$ correct, mean RT $864 \mathrm{~ms}$ ) and Dutch listeners without any knowledge of Cantonese or other tonal language $(92.6 \%$ correct, mean RT $845 \mathrm{~ms}$ ). In the present case, the unfamiliar Japanese items produced equivalent patterns from all three listener groups, too If anything, the Dutch control group slightly outdid the other groups on the hardest contrast (Figure 2), consistent with the tiny advantage they showed over the adoptees in processing the hardest Korean contrast also (Figure 1). The latter contrast (lenis vs. aspirated stops) was in fact hard for all participants. The adoptees and Dutch controls scored below chance level (39.7\% and $45.6 \%$, respectively) even at the post-test, and the Korean control participants had only $80.4 \%$ correct for these pairs, although they scored almost perfectly $(99.6 \%)$ for the other comparisons (fortis-lenis, fortis-aspirated, which were in fact quite distinguishable for all three groups). The acoustic cues distinguishing lenis from aspirated stops appear to be weak, making it a more difficult distinction even for native listeners, and supporting the contention that acoustic difficulty is the main determinant of discrimination task performance.

This is not to say that language-specific knowledge cannot be brought to bear if it is available and the task resembles familiar discrimination experience. In the present study, the Korean task was performed significantly better by the Korean controls, for whom the need to discriminate the stop contrasts is part of daily listening life. This advantage was apparent at all places of articulation and for all comparison types.

Note here that the 11 days of training in an identification task proved insufficient for the adoptees and Dutch control listeners to achieve native-like performance in discriminating Korean stops, although they (the adoptees especially) had certainly improved their identification performance across those 11 days. This suggests that the two task types are different enough for successful learning in one not to generalize to performance in the other (although it is an open question whether training with a discrimination task would affect identification performance, given that there is an acoustic-phonetic processing component in identification even though there need be no categorization component in discrimination). In future research, we would suggest that training adoptees and controls to enhance their perceptual sensitivity to the contrasts under study with a discrimination task should certainly lead to greater improvement at test of discrimination, and that it is then at least conceivable that adoptees might show better perceptual sensitivity than control listeners for their original language. Certainly the processing benefits so far reported for adoptees [e.g., 8, 9] seem to be best explained in terms of retained language knowledge. 


\section{References}

[1] E. A. Clark and J. Hanisee, "Intellectual and adaptive performance of Asian children in adoptive American settings,' Developmental Psychology, vol. 18, pp. 595-599, 1982.

[2] L. Isurin, "Deserted island or a child's first language forgetting," Bilingualism: Language and Cognition, vol. 3, pp. 151-166, 2000.

[3] E. Nicoladis and H. Grabois, "Learning English and losing Chinese: A case study of a child adopted from China," International Journal of Bilingualism, vol. 6, pp. 441-454, 2002.

[4] J. A. Roberts, K. E. Pollock, R. Krakow, J. Price, K. C. Fulmer, and P. P. Wang, "Language development in preschool-age children adopted from China," Journal of Speech, Language, and Hearing Research, vol. 48, pp. 93-107, 2005.

[5] K. A. Scott, J. A. Roberts, and R. Krakow, "Oral and written language development of children adopted from China,' American Journal of Speech-Language Pathology, vol. 17, pp. 150-160, 2008.

[6] C. Pallier, S. Dehaene, J.-B. Poline, D. LeBihan, A.-M. Argenti, E. Dupoux, and J. Mehler, "Brain imaging of language plasticity in adopted adults: Can a second language replace the first?" Cerebral Cortex, vol. 13, pp. 155-161, 2003.

[7] V. A. G. Ventureyra, C. Pallier, and H.-Y. Yoo,"The loss of firs language phonetic perception in adopted Koreans," Journal of Neurolinguistics, vol. 17, pp. 79-91, 2004

[8] J. Choi, "Rediscovering a forgotten language," Ph.D. dissertation, Radboud University Nijmegen, 2014.

[9] L. J. Pierce, D. Klein, J.-K. Chen, A. Delcenserie, and F. Genesee, "Mapping the unconscious maintenance of a lost first language," Proceedings of the National Academy of Sciences of the United States of America, vol. 111, pp. 17314-17319, 2014

[10] M. Broersma, D. Dediu, and J. Choi, "Individual differences in learning to perceive novel phonetic contrasts: How stable are they across time and paradigms?" Journal of the Acoustical Society of America, vol. 134, pp. 4107, 2013.

[11] E. Gerrits and M. E. H. Schouten, "Categorical perception depends on the discrimination task," Perception \& Psychophysics, vol. 66, pp. 363-376, 2004.
[12] M. Broersma, "Dutch listeners' perception of Korean fortis, lenis, and aspirated stops: First exposure," in Proceedings of the $6^{\text {th }}$ International Symposium on the Acquisition of Second Language Speech, New Sounds 2010, 2010, pp. 49-54.

[13] M. Sadakata and J. M. McQueen, "High stimulus variability in nonnative speech learning supports formation of abstract categories: Evidence from Japanese geminates," Journal of the Acoustical Society of America, vol. 134, pp. 1324-1335, 2013.

[14] M. Sonu, H. Kato, K. Tajima, R. Akahane-Yamada, and Y. Sagisaka, "Non-native perception and learning of the phonemic length contrast in spoken Japanese: training Korean listeners using words with geminate and singleton phonemes," Journal of East Asian Linguistics, vol. 22, pp. 373-398, 2013.

[15] T. J. Vance, An Introduction to Japanese Phonology. Albany, NY: State University of New York Press, 1987.

[16] H. Kubozono, J. Itô, and A. Mester, "Consonant gemination in Japanese loanword phonology," Proceedings of the $18^{\text {th }}$ International Congress of Linguists, pp. 953-973, 2008.

[17] D. McNicol, A Primer of Signal Detection Theory. Sydney: George Allen \& Unwin, 1972.

[18] C. Dietrich, D. Swingley, and J. F. Werker, "Native language governs interpretation of salient speech sound differences at 18 months," Proceedings of the National Academy of Sciences of the United States of America, vol. 104, pp. 16027-16031, 2007.

[19] T. K. Au, L. M. Knightly, S.-A. Jun, and J. S. Oh, "Overhearing a language during childhood," Psychological Science, vol. 13, pp. 238-243, 2002.

[20] L. M. Knightly, S.-A. Jun, J. S. Oh, and T. K. Au, "Production benefits of childhood overhearing," Journal of the Acoustical Society of America, vol. 114, pp. 465-474, 2003.

[21] J. S. Oh, S.-A. Jun, L. M. Knightly, and T. K. Au, "Holding on to childhood language memory," Cognition, vol. 86, pp. B53B64, 2003.

[22] R. C. Tees and J. F. Werker, "Perceptual flexibility: Maintenance or recovery of the ability to discriminate non-native speech sounds," Canadian Journal of Psychology, vol. 38, pp. 579-590, 1984.

[23] A. Cutler and H.-C. Chen, "Lexical tone in Cantonese spokenword processing," Perception \& Psychophysics, vol. 59, pp. $165-179,1997$. 